\title{
Guaranteed QoS Access by Packet-by-Packet Generalized Processor Sharing with Channel Errors and Long Propagation
}

\author{
Chia-Sheng Chang, Kwang-Cheng Chen \\ Institute of Communication Engineering, College of Electrical Engineering \\ National Taiwan University, Taipei, Taiwan, 10617, R.O.C.
}

\begin{abstract}
In this paper the PGPS/RAP multiaccess proposed in [16] is reinvestigated for realistic channel characteristics such as unreliable transmission and propagation delay effect. With our proposed link control scheme, the packet error rate of PGPS/RAP in an unreliable channel can be effectively improved while the packet delay for constant-bit-rate (CBR), variable-bit-rate (VBR) traffic is still guaranteed. Furthermore, with the technique of pipelining, we modify the PGPS/RAP to adapt the long propagation delay effect for guaranteed QoS access to CBR and VBR traffic of the endusers. This modified PGPS/RAP without any specific frame concept can still guarantee worst-case jitter of CBR and VBR traffic sources and therefore can guarantee the QoS of jittersensitive CBR and VBR sources.
\end{abstract}

\section{INTRODUCTION}

In [16], we demonstrated that Packet-by-Packet Generalized Processor Sharing [1]/Random addressed polling (PGPS/RAP) multiaccess is a very effective multiaccess approach in wireless multimedia networks with error-free channels and negligible propagation delay. However, wireless channels are inherently unreliable and time-varying compared to broadband, highly reliable, static wireline channels (e.g. coaxial cable or optical fiber channels). As a result, data link control schemes are definitely unavoidable to guarantee the Quality-of-Service (Q०S) of reliable information transmission in unreliable wireless channels. On the other hand, multimedia broadband networks may in general cover a wide geographical area and interconnect a huge number of end-user nodes. Because of the wide coverage, there often exists inevitable long propagation delay which may be multiple times of packet transmission duration in such networks. Propagation delay retards the information exchange between user nodes and is regarded as the primary degrading factor of most multiaccess protocol. Therefore, it is also a great challenge of designing multiaccess scheme with guaranteed QOS in multimedia broadband networks. The physical channels of these broadband networks often have the following two characteristics: 1) there are two separate subchannels. A broadcast channel conveys traffic from the access point to the end-users, and a multiaccess channel conveys traffic in the reversed direction, 2) a long propagation delay effect. Afterward, we refer to a channel with the above two characteristics as a long propagation delay channel.

This research was supported by the National Science Council of Tajwan, R.O.C. under the contract NSC-86-2221-E-007-035.

\section{PGPS/RAP MULTIACCESS}

\section{A. System Model}

Consider the following multiaccess communication model: There are $n$ nodes, each node has a queue of packets to be transmitted and the multiaccess channel is a common server (denote $C$ as the channel capacity). In addition, there is a special node called the coordinator where these $n$ nodes use this multiaccess channel to communicate with it. We try to serve three kinds of source: constant bit rate (CBR), variable bit rate (VBR), and available bit rate (ABR) sources.

We further assume: 1) Propagation delay is negligible compared to a packet transmission time. 2) All packets generated from a source are of the same size. 3) The packets of all ABR traffic sources are of the same size but the packet of CBR or VBR traffic sources may be of different size. 4) There exists a fixed parameter $L_{m a x}$, which is a size upper bound of all packets.

A CBR traffic source is characterized by four parameters $\left(r_{i}^{c}, L_{i}^{c}, T_{i}^{c}, D_{i}^{c}\right)$, where $r_{i}^{c}$ (bps) is the rate of this source, $L_{i}^{c}$ is the size of packets of this source, $T_{i}^{c}$ is the packet interarrival time, $D_{i}^{c}$ is the maximum tolerable delay measured from the arrival time of a packet to its departure time from the $i^{t h}$ CBR local queue, and $r_{i}^{c}=L_{i}^{c} / T_{i}^{c}$.

A VBR traffic source is characterized by five parameters $\left(r_{i}^{v}, L_{i}^{v}, T_{i}^{v}, Q_{i}^{v}, D_{i}^{v}\right)$, where $r_{i}^{v}$ (bps) is the peak rate of this source, $L_{i}^{v}$ is the size of packets of this source, $T_{i}^{v}$ is the minimum packet interarrival time, $Q_{i}^{v}$ is the query packet size of this source, $D_{i}^{v}$ is the maximum tolerable delay measured from the arrival time of a packet to its departure time from the $i^{\text {th }}$ VBR local queue, and $r_{i}^{v}=L_{i}^{v} / T_{i}^{v}$.

For $A B R$ source, we assume that if two or more $A B R$ sources send their packets in a transmission interval, then there is a collision. The packet is correctly received for only one packet in the multiaccess channel.

We adapt a simplified RAP protocol as the fundamental access. A complete operation of RAP can be found in [9].

Step 1 The base station broadcasts a [READY] message to all mobile nodes in its coverage area.

Step 2 Each active mobile node that intends to transmit generates a random number from the set $\{0,1,2, \ldots, p-1\}$ and simultaneously transmit their random numbers with orthogonal signals.

Step 3 The base station polls each active random number one by one. The mobile nodes who sent the polled random num- 
ber transmit packet to the base station.

Step 4 If the base station successfully/unsuccessfully receives the packet from any mobile node, it sends a positive/negative acknowledge ([PACK]/[NACK] $)$ right away before polling the next one(s). Then go back to Step 1.

A round of the above processes is called a polling cycle. From the point of view of $A B R$ nodes, the coordinator is just like a RAP base station. ABR nodes wait for [READY] signal to up-transmit random addresses and wait for the coordinator to poll each random address. The coordinator and ABR nodes are all aware of the following parameters: $p$ is the number of random addresses, and $L_{p}^{a}$ is the fixed ABR packet size. $P_{u}^{a}$ is the duration of time required for the coordinator to detect random addresses. $L_{u}^{a}$ is the equivalent packet size with transmission time $P_{u}^{a}$ in a channel with capacity $C$ bits per second, i.e. $L_{u}^{a} \equiv P_{u}^{a} \stackrel{u}{C}$.

It can be shown that for CBR and VBR nodes the packet delay is bounded under PGPS/RAP multiaccess [16]:

Proposition 1 (CBR delay bound) Denote $a_{p}$ the arrival time of a packet $p$ of the $i^{\text {th }}$ CBR source and $\hat{F}_{p}$ its departure time from the $i^{t h}$ CBR local queue. Under PGPS/RAP multiaccess, we have:

$$
\hat{F}_{p}-a_{p} \leq 2 L_{i}^{c} / r_{i}^{c}+L_{\max } / C
$$

Proposition 2 (VBR delay bound) Denote $a_{p}$ the arrival time of a packet $p$ of the $i^{t h}$ VBR source and $\hat{F}_{p}$ its departure time from the $i^{\text {th }}$ VBR local queue. Under PGPS/RAP multiaccess, suppose a query permit is generated every $N T_{i}^{v}$ seconds, we have:

$$
\hat{F}_{p}-a_{p} \leq N T_{i}^{v}+\frac{N T_{i}^{v}\left(L_{i}^{v}+Q_{i}^{v}\right)}{N L_{i}^{v}+Q_{i}^{v}}+\frac{L_{\max }}{C}
$$

\section{PGPS/RAP IN UnReliable ChanNels}

In the preceding discussion, the multiaccess channel is assumed to be reliable, i.e. without transmission error. Now we consider a more practical situation for multimedia wireless networks that the channel is fairly erroneous and a link control mechanism using retransmission is required to keep the packet error rate within an acceptable range. In this section, we propose the concept of retransmission permit to cope with the unreliable channel, and then analyze its impact on the delay bounds in [16].

A retransmission permit pattern corresponding to a CBR or VBR source is used to retransmit the erroneous packets, and is stated as follows:

1. CBR packet retransmission: A grant permit is generated at $t=n N T_{i}^{c}+L_{\max } / C, n=1,2, \ldots$, in case that there is any packet error in the previous $N$ transmissions. The PGPS weighting factor of this permit queue is set to $r_{i}^{c} / N$ and the total bandwidth $\phi_{i}^{c}$ allocated to this CBR source is $r_{i}^{c}(1+1 / N)$.

2. VBR packet retransmission: A grant permit is generated at $t=n N T_{i}^{v}+L_{\max } / C, n=1,2, \ldots$, in case that any packets which arrive in the $n^{\text {th }}$ counting interval (i.e. $\left.\left[(n-1) N T_{i}^{v}, n N T_{i}^{v}\right)\right)$ is erroneously transmitted. The PGPS weighting factor of this permit queue is set to $r_{i}^{v} / N$ and the total bandwidth $\phi_{i}^{v}$ allocated to this VBR source is $r_{i}^{v}\left(1+\frac{1}{N}+\frac{Q_{i}^{v}}{N L_{i}^{v}}\right)$.

Whenever the coordinator selects a retransmission grant permit to serve, it notifies the corresponding CBR or VBR source to retransmit the packet in error.

Suppose a CBR or VBR packet-error occurs independently with probability $p$, then the packet transmissions can be seen as a sequence of Bernoulli trials. Hence the expected number of erroneous packets in $N$ transmissions is $N p$. With this retransmission mechanism, a retransmission opportunity is provided every $N$ transmissions and the expected number of erroneous packets in $N$ trials is reduced to $N(N+1) p^{2} / 2$ provided $p \ll 1$ (see Appendix A of [15]). Therefore by adjusting the value of $N$, we can reach an acceptable packet error rate to meet QoS in multimedia wireless networks.

It can be shown that for CBR and VBR nodes the packet delay is bounded under PGPS/RAP multiaccess provided that $\sum \phi_{i}^{c}+\sum \phi_{i}^{v}<C[15]$ :

Proposition 3 (CBR delay bound with retransmission) Sup pose a retransmission opportunity is provided every $N T_{i}^{c}$ seconds, and denote $a_{e}$ the arrival time of an erroneous packet $e$ of the $i^{t h}$ CBR source and $\hat{F}_{r}$ its retransmission departure time from the $i^{\text {th }}$ CBR local queue. Then we have:

$$
\hat{F}_{r}-a_{e} \leq(2 N+1) \cdot L_{i}^{c} / r_{i}^{c}+2 L_{\max } / C
$$

Proposition 4 (VBR delay bound with retransmission) Sup pose a retransmission opportunity is provided every counting interval, and denote $a_{e}$ the arrival time of an erroneous packet $e$ of the $i^{t h}$ VBR source and $\hat{F}_{T}$ its retransmission departure time from the $i^{\text {th }}$ VBR local queue. Then we have:

$$
\hat{F}_{r}-a_{e} \leq 3 N \cdot L_{i}^{v} / r_{i}^{v}+2 L_{\max } / C
$$

From the discussion above, it can be seen that with retransmission CBR and VBR delay bounds are enlarged. The primary contributions to these enlargement are the waiting time intervals for retransmissions. Besides, the retransmission permit certainly occupy additional channel bandwidth. However, due to diversed QoS demands, this mechanism is only implemented while is needed.

\section{PGPS/RAP Multiaccess in a Long Propagation Delay Channel}

\section{A. Long Propagation Delay Channel Model}

Consider a long propagation delay channel mentioned in Section I. In this channel there are $n$ user nodes, each of which has a queue of packets to be transmitted. A special node called the coordinator, which is the access point of this network, uses the broadcast channel (with bandwidth $C_{B}$ ) to communicate with other nodes and all user nodes use the multiaccess channel (with bandwidth $C_{U}$ ) to communicate with the coordinator.

To model the propagation delay effect, every signal travels at a constant speed $V$. A CBR traffic source is now characterized by five parameters $\left(r_{i}^{c}, L_{i}^{c}, T_{i}^{c}, J_{i}^{c}, \tau_{i}^{c}\right)$, where $r_{i}^{c}$ is the rate of this source, $L_{i}^{c}$ is the size of packets of this source, $T_{i}^{c}$ is the packet interarrival time, $J_{i}^{c}$ is the maximum tolerable jitter of this source (jitter is defined as the difference between 
the time of two successive departures and the time of two successive arrivals [10]), and $\tau_{i}^{c}$ represents the propagation time needed for a signal to travel from the coordinator to this user (or the propagation time in the reverse direction).

A VBR traffic source is now characterized by six parameters $\left(r_{i}^{v}, L_{i}^{v}, T_{i}^{v}, Q_{i}^{v}, J_{i}^{v}, \tau_{i}^{v}\right)$, where $r_{i}^{v}$ is the peak rate of this source, $L_{i}^{v}$ is the packet size of packets of this source, $T_{i}^{v}$ is the minimum packet interarrival time, $Q_{i}^{v}$ is the query packet size of this source, and $J_{i}^{v}, \tau_{i}^{v}$ represent the maximum tolerable jitter and propagation time, respectively. In addition, we suppose that:

1. If a packet overlaps in time-axis with any other packets when it arrives at the coordinator, then there is a collision and the coordinator obtains no information about the contents or source of the transmitted packets. If just one packet arrives at the coordinator without any overlapping with other packets, the packet is correctly received.

2. There is a fixed parameter $\tau_{\max }$, which is an upper bound of propagation delays from all user nodes.

3. Signaling packets issued from the coordinator are small compared to packets from other user nodes. Therefore such signaling packets are assumed to be negligible.

\section{B. RAP Protocol with Long Propagation Delay}

The operation of RAP protocol has been stated in Section II. However, in a long propagation delay channel, it takes time for the random address signal and data packet signal to travel between ABR users and the coordinator. If the coordinator just sends out a [READY] signal and keeps idly waiting for the random address signal to propagate back, the portion of time used to execute RAP protocol will be low to yield poor efficiency. To overcome this problem, we use the technique known as pipelining. With pipelining, the RAP protocol is modified as follows:

(i) Initially the coordinator sends out a READY signal to start a polling cycle.

(ii) The coordinator checks if there are any random address signals returning already. If this is the case, it polls each active random address one by one. Otherwise it sends out another READY signal to initiate another polling cycle.

(iii) Goto (ii)

In this way, multiple RAP polling cycles are pipelined together and this takes advantage of the waiting time induced by propagation delay. Therefore this modification maintains high efficiency in a long propagation channel.

With pipelining there are also modifications on the ABR user side. Every ABR user has three queues locally. They are called polling queue, collision queue, and new arrival queue, respectively. Every packet stored in polling is marked with a random address, and waiting for polling signal from the coordinator. If a packet from polling queue collides with packets of other ABR users, this packet will be forwarded to collision queue and waiting for re-polling there. Whenever a ABR user hears a READY signal from the coordinator,

(i) it checks if there are any packets stored in collision queue.

(i.1) if yes, it gets one packet from the collision queue.

(i.2) otherwise it checks if there are any packets stored in new arrival queue. (i.2.1) if yes, it gets one packet from new arrival queue.

(i.2.2) otherwise go to (iii).

(ii) the ABR user generates a random address, and uptransmits this random address via the multiaccess channel. Then it marks this random address on the packet we got in (i), and finally put this packet in polling queue.

(iii) End.

Because all users have different propagation parameters $\tau_{i}^{a}$, to make the response signals (random addresses signal or ABR packets signal) of different ABR users coincide, and to "normalize" the round trip delay of CBR or VBR source to $2 \tau_{\max }$, all users transmit packets into the multiaccess channel with proper delay after they receive signals from the coordinator. Therefore we propose that for any user $i$, it issues its response signal into the multiaccess channel with delay $2 \times\left(\tau_{\max }-\tau_{i}\right)$ seconds after it hears the signal from the coordinator. With this proper delay, response signals of different ABR users coincide with each other in the multiaccess channel.

\section{PGPS/RAP with Propagation Delay}

As earlier discussion, permits according to presumed patterns are generated in the coordinator.

1. For a $\left(r_{i}^{c}, L_{i}^{c}, T_{i}^{c}, J_{i}^{c}, \tau_{i}^{c}\right)$ CBR source, a grant permit with size, $L_{i}^{c}$ is generated every $T_{i}^{c}$ seconds.

2. For a $\left(r_{i}^{v}, L_{i}^{v}, T_{i}^{v}, Q_{i}^{v}, J_{i}^{v}, \tau_{i}^{v}\right)$ VBR source, a query permit with size $Q_{i}^{v}$ is generated every $N T_{i}^{v}$ seconds, where $N$ is the maximum positive integer satisfying: $J_{i}^{v} \geq N T_{i}^{v}+\frac{N L_{i}^{v} T_{i}^{v}}{N L_{i}^{v}+Q_{i}^{v}}+$ $\frac{L_{\max }-L_{i}^{\nu}}{C_{U}}$. Denote $K$ the minimum positive integer such that $K N T_{i}^{v}>2 \tau_{\text {rnax }}+\frac{N Q_{i}^{v} T_{i}^{v}}{N L_{i}^{v}+Q_{i}^{v}}+\frac{L_{\max }-Q_{i}^{v}}{C_{U}}$, which represents the minimum number of pipelined query operations needed to fill up the round-trip propagation delay. Whenever a query permit is chosen to serve by the coordinator, the following tasks are performed: 1 ) the coordinator queries the $i^{t h} \mathrm{VBR}$ user the number of packet arrivals at the $i^{\text {th }} \mathrm{VBR}$ local queue in the $n^{\text {th }}$ counting interval, $\left.\left[(n-1) N T_{i}^{v}, n N T_{i}^{v}\right) .2\right)$ the $i^{t h}$ VBR user receives this query and replies the number. Let $n$ be the result of the query, then

(a) if $n=0$, no grant permits are generated.

(b) if $n>0$, a grant permit with length $L_{i}^{v}$ is generated $K N T_{i}^{v}+N T_{i}^{v} Q_{i}^{v} /\left(N L_{i}^{v}+Q_{i}^{v}\right)$ seconds after this query permit. Then another is generated every $N T_{i}^{v} L_{i}^{v} /\left(N L_{i}^{v}+Q_{i}^{v}\right)$ seconds until all $n$ grant permits are generated.

3. Unlike CBR and VBR users, each has its own corresponding presumed arrival pattern, there is only one presumed arrival pattern for all ABR users. A permit determines its successors as follows: According to our RAP operation with pipelining in Section IV-B,

(a) The first permit is a RA permit with size $L_{u}^{a}$. The corresponding operation is that the coordinator broadcasts [READY] and detects active Random Addresses.

(b) The coordinator checks if there are any random address signals returning back. If yes, the next permits are polling permits with size $L_{p}^{a}$, which represent polling each active address one by one. Otherwise, the next permit is another RA permit. 
(c) Goto (b).

Given the modified permit generation mechanism, the PGPS/RAP protocol with long propagation delay is stated as follows:

(i) Given the modified permit generating mechanisms stated above, set the weighting factor $\phi_{i}^{c}$ of the $i^{t h}$ CBR source to $r_{i}^{c}$, set the weighting factor $\phi_{i}^{v}$ of the $i^{t h}$ VBR source to $r_{i}^{v} \times$ $\left(1+\frac{1}{N} \cdot \frac{Q_{i}^{v}}{L_{i}^{v}}\right)$ and set the weighting factor $\phi^{a}$ of the ABR queue to $C_{U}-\sum_{i} \phi_{i}^{c}-\sum_{i} \phi_{i}^{v}$.

(ii) Use a PGPS scheduler to select a permit queue and then get one permit from the selected queue. Denote $L$ (bits) the packet size of this permit.

(ii.1) if this permit is for the $i^{\text {th }} \mathrm{CBR}$ user, the coordinator issues a notification signal into broadcast channel to notify the $i^{\text {th }}$ CBR user to transmit a packet.

(ii.2) if this permit is for the $i^{t h}$ VBR user, and

(ii.2.1) it is a query permit, the coordinator issues a query signal into the broadcast channel to query the $i^{\text {th }}$ VBR user. (ii.2.2) it is a grant permit, the coordinator issues a notification signal into the broadcast channel to notify the $i^{t h}$ VBR user to transmit a packet.

(ii.3) if this permit is from the $\mathrm{ABR}$ queue, and

(ii.3.1) it is a RA permit, the coordinator issues a READY signal into the broadcast channel to ask all ABR users to up-transmit random addresses.

(ii.3.2) it is a polling permit, the coordinator polls the next un-polled random address by issuing a polling signal into the broadcast channel.

(iii) Wait for $L / C_{U}$ seconds and go to (ii).

With the above operations, it can be shown that for CBR and VBR sources the packet jitter is bounded. We present the bounds in two propositions, while proofs are in [15].

Proposition 5 (CBR jitter bound) Let $D_{p}$ and $\hat{D}_{p}$ denote the interarrival time and the interdeparture time of packet $p$ and packet $p+1$ of a CBR source. In a channel with propagation delay upper bound $\tau_{m a x}$ and under PGPS/RAP protocol, we have:

$$
\left|\hat{D}_{p}-D_{p}\right| \leq \frac{L_{i}^{c}}{r_{i}^{c}}-\frac{L_{i}^{c}}{C_{U}}+\frac{L_{\max }}{C_{U}}
$$

Proposition 6 (VBR jitter bound) Let $D_{p}$ and $\hat{D}_{p}$ denote the interarrival time and the interdeparture time of packet $p$ and packet $p+1$ of a VBR source. In a channel with propagation delay upper bound $\tau_{\max }$ and under PGPS/RAP protocol, suppose a query permit is generated every $N T_{i}^{v}$ seconds, then we have:

$$
\left|\hat{D}_{p}-D_{p}\right| \leq N T_{i}^{v}+\frac{N L_{i}^{v} T_{i}^{v}}{N L_{i}^{v}+Q_{i}^{v}}+\frac{L_{\max }-L_{i}^{v}}{C_{U}}
$$

\section{Simulation Results}

In this section, we give some simulation results of PGPS/RAP multiaccess with various CBR and VBR traffic sources in multiaccess channels with different capacities.

In case 1 and case 2 , we simulate the maximal delay of three CBR and four VBR traffic sources without and with retransmission mechanisms and compare the results of these two situations. In case 1 , we simulate the maximal delays of three
CBR and four VBR traffic sources in a channel with capacity $25 \mathrm{Mbps}$. The remaining bandwidth is used to execute RAP operation with parameters $p=5, L_{u}^{a}=200, L_{p}^{a}=1000$. The four VBR sources are packetized output of MPEG-1 video coder with the famous movie "star war" as its input. The detailed traffic parameters of the sources, the theoretical delay bound and maximal delays in simulation are listed in Table I. To compare the effect of retransmissions, in case 2 we consider the same traffic sources as in case 1 but retransmission link control mechanisms are exerted for all CBR and VBR sources over a channel with packet error rate $10^{-2}$. For example, a retransmission opportunity is provided for every 5 CBR 1 transmissions. Note that the bandwidth of each traffic source is increased to accommodate additional retransmission traffic. The detailed traffic parameters of all traffic sources, the theoretical delay bounds and maximal delays in simulation are listed in Table II. From the results of case 1 and case 2 , at the expected price of larger maximal delays, retransmissions of erroneous packets can improve the packet error rate from $10^{-2}$ to the order of $10^{-4}$ to meet the required packet error rate. Therefore this simple link control mechanism can effectively provide more reliable PGPS/RAP access over an erroneous channel.

In case 3 , we simulate the maximal jitter of ten CBR and seven VBR traffic sources in a $10 \mathrm{Mbps}$ channel with maximal delay $\tau_{\text {max }}=0.01$ second. The VBR source 1 is the output of a four-rate-level packet generator. The generator stays in each rate level for 0.015 seconds, and randomly decides the next rate level with equal probabilities. The four rate levels are: $0,180 \mathrm{kbps}, 270 \mathrm{kbps}, 540 \mathrm{kbps}$. Other VBR sources are outputs from ON-OFF coders. For instance, VBR source 2 generates a packet of size 390 bits every 0.001 second with probability 0.6 . The CBR and VBR traffic sources take up $98 \%$ of the channel capacity and the remaining $2 \%$ are used for ABR service, with the parameter $p=5, L_{p}^{a}=1000$, $L_{u}^{a}=200$. The details of all traffic sources are listed in Table III. The simulation maximum jitter v.s. the theoretical jitter bounds are listed in Table IV $\left(C=10^{7}, L_{\max }=1000\right.$, $\tau_{\max }=0.01$ ). Although only $2 \%$ of total bandwidth is originally allocated to ABR traffic, approximately $10 \%$ of time are flexibly used to execute RAP to provide ABR service. ABR traffic indeed takes advantage of the surplus bandwidth when VBR source are in their lower-rate period. This result justifies our jitter bounds and demonstrates the effectiveness of PGPS/RAP multiaccess in networks with long propagation delay.

\section{Conclusions}

In this paper, we first proposed a link control mechanism for PGPS/RAP such that this multiple access methodology with guaranteed QoS can operate in unreliable wireless channels.

With the modification according to pipelining concept, we also proposed PGPS/RAP methodology as an effective solution for the multiaccess scheme to provide broadband network access services to the end-users. With pipelining, multiple RAP operations and multiple VBR query operations can be pipelined together to take advantage of the propagation 
time and thus this modification maintains high efficiency of PGPS/RAP in such a broadband access channel.

Except for difference required to adapt unreliable transmission and propagation effect, the above modified PGPS/RAP inherits many good characteristics from original version [16]. As a result, PGPS/RAP is not only a potential multiaccess approach for wireless multimedia networks, but also an competent access protocol in broadband access networks with more general topology and with long propagation delay effects.

\section{ACKNOWLEDGMENT}

The authors appreciate Professor Cheng-Shang Chang's helpful discussions, who is with the National Tsing Hua University, Taiwan.

\section{REFERENCES}

[1] A. K. Parekh, R. G. Gallager, "A Generalized Processor Sharing Approach to Flow Control in Integrated Services Networks: The Single-Node Case," IEEE/ACM Transactions on Networking, Vol. 1, No. 3, pp. 344-357, June 1993.

[2] T. Zahariadis, et al., "Interactive Multimedia Services to Residential Users," IEEE Communications Magazine, June, 1997.

(3] Leif Aarthun Ims, et al., "Interactive Digital Video Networks: Lessons from a Commercial Deployment," IEEE Communications Magazine, June, 1997.

(4) Carel-Jan L. van Driel, et al., "The (R)evolution of Access Networks for the Information Superhighway" IEEE Communications Magazine, June, 1997.

[5] T. Koonen, et al., "TOBASCO: An Innovative Approach for Upgrading CATV Fiber-Coax Networks for Broadband Interactive Services," IEEE Communications Magazine, Apr. 1997.

6] D. J. Goodman, R. A. Valenzuela, K. T. Gayliard, and B. Ramamurthi, "Packet reservation multiple access for local wireless communications," IEEE Trans. Commun., vol. 37, pp. 885-890, Aug. 1989.

[7] G. Anastasi, D. Grillo, L. Lenzini, "An Access Protocol for Speech/Data/Video Integration in TDMA-Based Advanced Mobile Systems," IEEE Journal on Selected Areas in Comm., Vol. 15, No. 8, 1997.

[8] L. Kleinrock, F. A. Tobagi, "Packet Switching in Radio Channels: Part I-Carrier Sense Multiple Access Modes and Their Throughput Delay Characteristics," IEEE Trans. on Communications, vol. COM-23, no. 12, pp. 14001416, Dec. 1975.

[9] Kwang-Cheng Chen, Cheng-Hua Lee, "RAP-A Novel Medium Access Control Protocol for Wireless Data Networks," Proc. IEEE Globecom'93, Houston, 1993.

[10] Cheng-Shang Chang, Kwang-Cheng Chen, Ming-Young You, JinFu Chang, "Guaranteed Quality-of-Service Wireless Access to ATM," IEEE Journal on Selected Areas in Communications, vol. 15 , no. 1, pp. 106-118, 1997.

[11] Y. K. Sun, K. C. Chen, D. C. Twu, "Multiple Access in Packet Switching Networks: Part I-Generalized Tree Protocol," submitted for publication.

[12] D. C. Twu, K. C. Chen, Y. K. Sun, "Multiple Access in Packet Switching Networks: Part II-Stability Properties," submitted for publication.

[13] K. C. Chen, "Multiple Access for Wireless Packet Networks," Multiple Access, Mobility, and Traffic for Personal Comm. Workshop, 1997.

[14] Chia-Sheng Chang, Kwang-Cheng Chen, "Guaranteed Quality-ofservice Medium Access by Packet-by-Packet Generalized Processor Sharing Algorithm: Part I-Multimedia Radio," submitted for publication.

[15] C. S. Chang, K. C. Chen "Guaranteed Quality-of-Service Medium Access by Packet-by-Packet Generalized Processor Sharing Algorithm: Part II-Broadband Access," submitted for publication.

[16] Chia-Sheng Chang, Kwang-Cheng Chen " Guaranteed Quality-ofService Wireless Medium Access by Packet-by-Packet Generalized Processor Sharing Algorithm," Proc. IEEE ICC'98, Atlanta, 1998.
TABLE I

CBR AND VBR SOURCES AND SIMULATION RESULT (CASE 1).

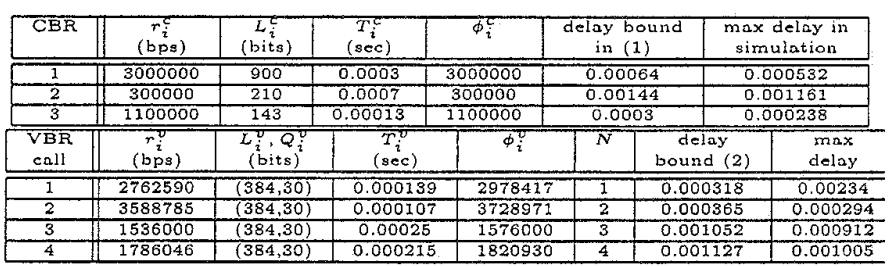

TABLE II

CBR AND VBR SOURCES AND SIMULATION RESULT (CASE 2).

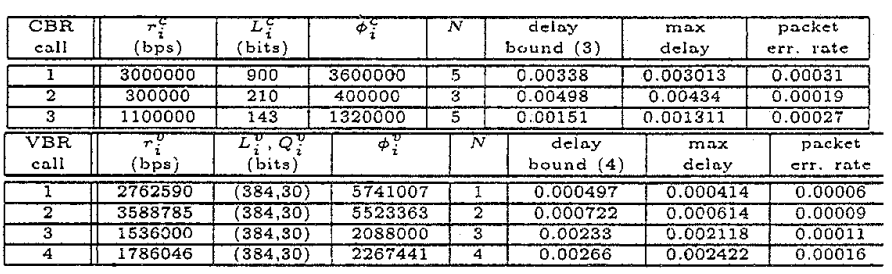

TABLE III

Simulation traffic sources (CASe 3).

\begin{tabular}{|c||c|c|c|c|c|c|}
\hline call & $\begin{array}{c}r_{i}^{c}, r_{i}^{\nu} \\
(\mathrm{bps})\end{array}$ & $\begin{array}{c}L_{i}^{c},\left(L_{i}^{\nu}, Q_{i}^{v}\right) \\
(\mathrm{bits})\end{array}$ & $p_{o n}$ & $\phi_{i}^{c}, \phi_{i}^{v}$ & $\begin{array}{c}N T_{i}^{v} \\
(\mathrm{sec} .)\end{array}$ & $\begin{array}{c}T_{i}^{c}, \tau_{i}^{v} \\
(\mathrm{sec})\end{array}$ \\
\hline CBR1 & 2000000 & 500 & N/A & 2000000 & N/A & 0.001 \\
\hline CBR2 & 1500000 & 900 & N/A & 1500000 & N/A & 0.002 \\
\hline CBR3 & 1000000 & 600 & N/A & 1000000 & N/A & 0.003 \\
\hline CBR4 & 850000 & 170 & N/A & 850000 & N/A & 0.004 \\
\hline CBR5 & 750000 & 240 & N/A & 750000 & N/A & 0.005 \\
\hline CBR6 & 600000 & 480 & N/A & 600000 & N/A & 0.006 \\
\hline CBR7 & 500000 & 450 & N/A & 500000 & N/A & 0.007 \\
\hline CBR8 & 250000 & 200 & N/A & 250000 & N/A & 0.008 \\
\hline CBR9 & 250000 & 100 & N/A & 250000 & N/A & 0.009 \\
\hline CBR10 & 100000 & 700 & N/A & 100000 & N/A & 0.01 \\
\hline VBR1 & 540000 & $(270,30)$ & N/A & 600000 & 0.0005 & 0.001 \\
\hline VBR2 & 390000 & $(390,30)$ & 0.6 & 400000 & 0.003 & 0.002 \\
\hline VBR3 & 280000 & $(350,50)$ & 0.8 & 300000 & 0.0025 & 0.003 \\
\hline VBR4 & 280000 & $(490,70)$ & 0.5 & 300000 & 0.0035 & 0.004 \\
\hline VBR5 & 180000 & $(450,50)$ & 0.7 & 200000 & 0.0025 & 0.005 \\
\hline VBR6 & 96000 & $(240,40)$ & 0.2 & 100000 & 0.01 & 0.006 \\
\hline VBR7 & 90000 & $(360,40)$ & 0.4 & 100000 & 0.004 & 0.007 \\
\hline
\end{tabular}

TABLE IV

SiMULATION RESULTS V.S. THEORETICAL BOUNDS IN (5) (6) (CASE 3 ).

\begin{tabular}{|c||c|c|c||c|c|}
\hline call & $\begin{array}{c}\text { max. jitter } \\
\text { (sec.) }\end{array}$ & $\begin{array}{c}\text { bound } \\
\text { (sec.) }\end{array}$ & call & $\begin{array}{c}\text { max. jitter } \\
\text { (sec.) }\end{array}$ & $\begin{array}{c}\text { bound } \\
\text { (sec.) }\end{array}$ \\
\hline \hline CBR1 & 0.000131 & 0.0003 & CBR10 & 0.002936 & 0.00703 \\
\hline CBR2 & 0.000284 & 0.00061 & VBR1 & 0.000213 & 0.001023 \\
\hline CBR3 & 0.000363 & 0.00064 & VBR2 & 0.002243 & 0.004036 \\
\hline CBR4 & 0.000136 & 0.000283 & VBR3 & 0.001749 & 0.003731 \\
\hline CBR5 & 0.000192 & 0.000369 & VBR4 & 0.002377 & 0.005184 \\
\hline CBR6 & 0.000441 & 0.000852 & VBR5 & 0.00123 & 0.004805 \\
\hline CBR7 & 0.0006 & 0.000955 & VBR6 & 0.008317 & 0.012467 \\
\hline CBR8 & 0.000503 & 0.00088 & VBR7 & 0.001591 & 0.007664 \\
\hline CBR9 & 0.000203 & 0.00049 & & & \\
\hline
\end{tabular}

